\title{
Effectiveness and safety of topical tacrolimus monotherapy for repigmentation in vitiligo: a comprehensive literature review ${ }^{*}$
}

\author{
Andrea Sisti ${ }^{1}$ \\ Giovanni Sisti ${ }^{2}$ \\ Carlo Maria Oranges ${ }^{3}$
}

DOI: http:/ / dx.doi.org/10.1590/abd1806-4841.20164012

\begin{abstract}
Thus far, several small studies and case reports on the use of topical immunomodulators in vitiligo have been published. We undertook a comprehensive literature review, searching for studies evaluating clinical response to tacrolimus topical therapy for vitiligo. A search was performed on PubMed/Medline using the term "vitiligo", combined with "topical" and "ointment". Our inclusion criteria were: use of tacrolimus ointment as monotherapy to treat vitiligo. We found 29 studies from 2002 to 2014. Overall, 709 patients were treated in 29 studies. Pooling the lesions, $50 \%$ repigmentation of vitiligo patches was never achieved before 2 months of treatment, with a peak after 6 months of therapy. The best results were obtained on lesions of the cephalic region, especially the face, with tacrolimus $0.1 \%$ ointment two times daily. The percentage of non-responsive patients ranged from $0 \%$ to $14 \%$. Treatment was generally well-tolerated; only localized adverse effects were reported. Our objective was to verify the effectiveness and safety of tacrolimus ointment monotherapy. It has good efficacy and tolerability. At present, only small trials and case series are available in the literature. Further, standardized investigations on a larger number of patients are needed.
\end{abstract}

Keywords: Ointments; Tacrolimus; Vitiligo

\section{INTRODUCTION}

Vitiligo is characterized by the progressive disappearance of melanocytes, resulting in depigmentation of the skin and/or hair. The etiology of vitiligo is unknown. ${ }^{1}$ Genetic studies support a non-Mendelian inheritance, suggesting that vitiligo is a multifactorial, polygenic disorder. The autoimmune theory remains the most widely accepted. Vitiligo has frequently been reported in association with autoimmune disorders such as thyroid disease, diabetes mellitus and alopecia areata.

Several studies have suggested that the presence of increased antimelanocyte antibodies and the imbalance of T-cell (CD4+/CD8+ and Tregs) subsets, along with their functional defects, may result in melanocyte destruction in vitiligo patients. ${ }^{2}$

The disease affects both genders equally. It can appear at any age and the average age of onset is somewhat variable in different geographic regions. The mean onset age is reportedly 22 in the U.S. and India, 24 in Brazil and 25 in the UK. ${ }^{3}$
Vitiligo treatment remains a challenge. Therapeutic options for vitiligo include: topical and systemic corticosteroids, topical calcineurin inhibitors, calcipotriol, phototherapy, excimer laser, and surgical methods such as skin/single-hair grafting, autologous cultured melanocyte or epidermal suspension transplantations.

Topical corticosteroids are most commonly used drug to treat vitiligo but there are concerns over side effects due to long-term use. Steroid application causes skin atrophy, telangiectasia, hypertrichosis and acne.

Tacrolimus and pimecrolimus are used as topical immunomodulators. They inhibit calcineurin action, thus preventing T-cell activation and the production of various inflammatory cytokines.

Both have been used to treat other inflammatory and immunologic skin disorders, including vitiligo, with encouraging results. ${ }^{4,5}$

Tacrolimus is a macrolide antibiotic produced

Received on 08.09.2014

Approved by the Advisory Board and accepted for publication on 14.10.2014

Work performed in Montecatini Terme - Pistoia, Italy.

Financial Support: None.

Conflict of Interest: None.

Montecatini Terme - Pistoia, Italy

Department of Health Sciences, University of Florence, AOU Careggi - Florence, Italy

Marche Polytechnic University - Ancona, Italy.

(C2016 by Anais Brasileiros de Dermatologia 
by Streptomyces tsukubaensis with strong T-specific, immunosuppressant activity. The biological activity of tacrolimus takes effect after binding to the cytosolic 12$\mathrm{kd}$ macrophilin FK506 binding protein (FK-BP). The tacrolimus/FK-BP complex inhibits calcineurin-mediated phosphorylation of the transcription factor, the nuclear factor of activated T-cells (NFAT). Hence, the expression of several inflammatory T-cell cytokines is inhibited.

Indeed, topical tacrolimus downregulates proinflammatory cytokines, namely IL-2, IL-3, IL4, IL-5, IFN-c, TNF-a and granulocyte-stimulating factors. Lan et al. $^{6}$ reported that the proliferation of melanocytes was significantly enhanced by tacrolimus-treated keratinocyte supernatant. Further, they noted that the concentration of stem-cell factor and matrix metallopeptidase-9 activity in tacrolimus-treated keratinocyte supernatant increased significantly. They suggested that their results provided in vitro evidence demonstrating the positive effect of tacrolimus on melanocyte growth and migration.

Thus far, several small studies and case reports on the use of topical immunomodulators in vitiligo have been published.

\section{METHODS}

To verify the effectiveness of tacrolimus alone, we selected studies that discussed tacrolimus ointment as monotherapy for vitiligo treatment.

A literature search on Medline/PubMed was performed for articles evaluating clinical response using tacrolimus as topical therapy for vitiligo. The keywords were: "vitiligo" combined with "topical" and "ointment".

Inclusion criteria were: 1) case study, review of literature, case report, clinical trial, open-label prospective study 2) tacrolimus used as monotherapy. The exclusion criterion was tacrolimus as a combination therapy.

The entire PubMed database was explored, without time restrictions. Each article was tabulated as follows: authors, year of study, type of study, number of patients, age (in years) and sex of patients, localization of disease, treatment protocol, adverse effects, outcome.

Studies discussing children and adults were included, along with studies describing topical treatment with both tacrolimus ointment $0.03 \%$ and tacrolimus ointment $0.1 \%$. English and non-English-language papers were included.

The publications were screened manually and reviewed to identify reports on tacrolimus monotherapy. Three investigators independently reviewed and extracted data from the papers according to the predetermined criteria.

RESULTS
We identified 117 full-text articles; 88 did not meet the inclusion criteria, leaving 29 studies available from 2002 to 2014. Nineteen were open-label trials, 3 were retrospective cohorts, 6 were case reports and 1 was a case series (Chart 1 ).

Overall, the treatments of 709 patients were described in 29 studies. The main treatment choice was tacrolimus ointment $0.1 \%$, applied twice daily (19/29 studies, $65 \%$ ) and once daily in 7 studies. Six studies examined treatment with tacrolimus $0.03 \%$ once daily. ${ }^{7-12}$

Treatment length was variable, with a mean duration of 5.2 months (ranging from 2 to 18 months). All patients were advised to use sunscreen regularly and avoid intentional sun exposure during the day.

Response rates also varied. Pooling the lesions, $50 \%$ repigmentation of vitiligo patches was never achieved before 2 months of vitiligo treatment, with a peak after 6 months of therapy. ${ }^{13}$ In all responder-patients undergoing treatment regimens, at least $50 \%$ repigmentation was achieved after 6 months of therapy. The best results were obtained on lesions in the cephalic regions, especially the face, applying tacrolimus ointment twice daily. ${ }^{14-16}$

The percentage of non-responsive patients ranged from $0 \%$ to $14 \%$. Only Kathuria et al. reported unsatisfactory results and limitations to the drug, with $50 \%$ repigmentation after 6 months of therapy in 5.3\% of patients. ${ }^{17}$

Treatment was generally well-tolerated; no adverse systemic effects were reported. The most frequent adverse effects were burning sensation and pruritus, local erythema or irritation, acne or folliculitis-like manifestations, dysesthesia, stinging, pickling, formication and soreness. ${ }^{7,14,15,18-27}$

\section{DISCUSSION}

Our review aimed to critically assess the studies evaluating monotherapy with tacrolimus ointment to treat vitiligo.

Selection bias and a lack of common outcome measures were among the issues that prevented a proper meta-analysis. Although this review is not a meta-analysis, we critically assessed the literature and tried to identify high-quality studies.

The main limits of this analysis are the low number of patients included in most studies and the high heterogeneity of the study populations. Nevertheless, the studies analyzed as a whole seem to show that tacrolimus ointment provides effective treatment. Substantial repigmentation of lesions $(>50 \%)$ requires time and consistent application of the product, at least 2-3 months depending on the patient's age and race. ${ }^{28,29,30}$

Treatment with topical tacrolimus is generally 


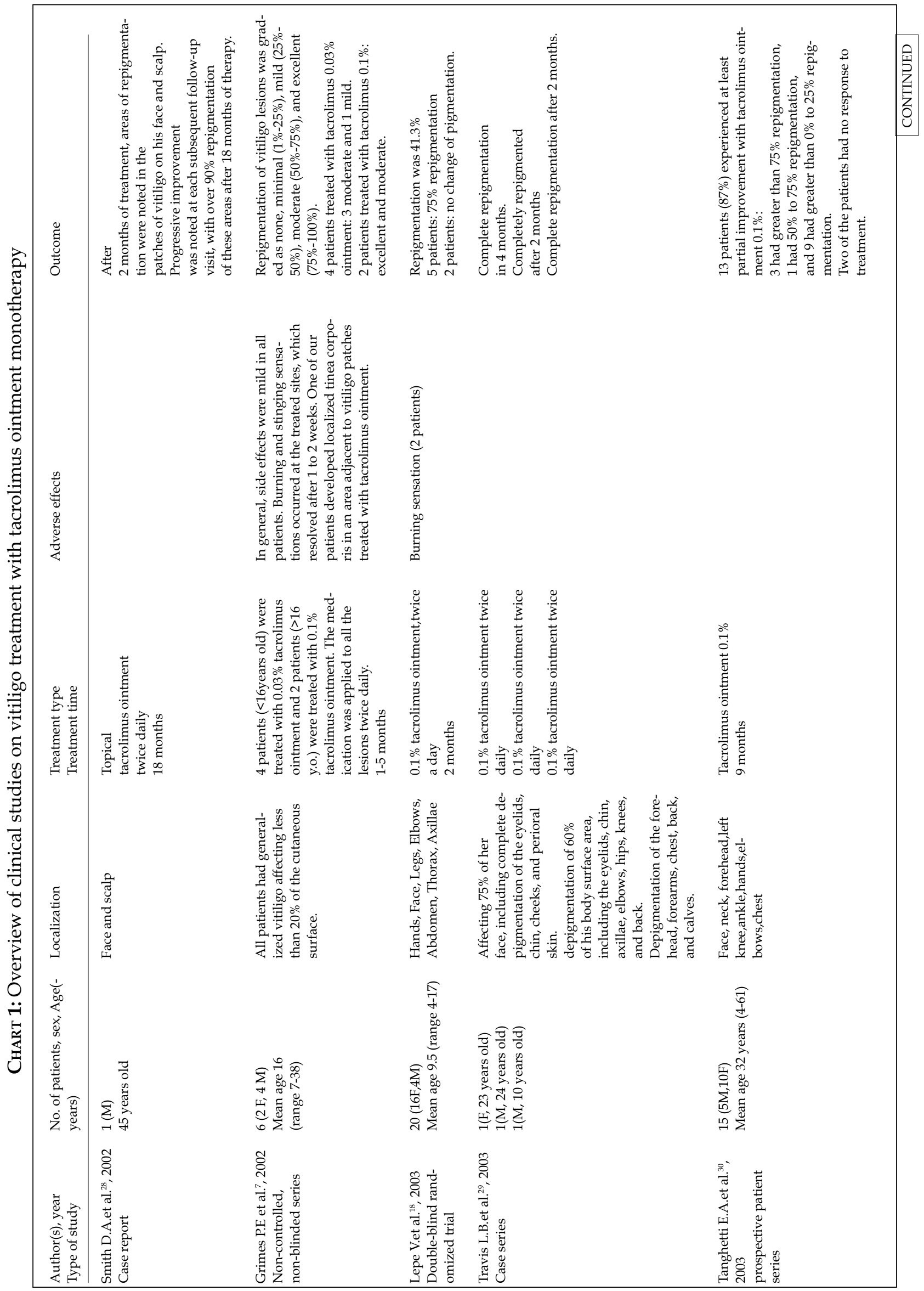




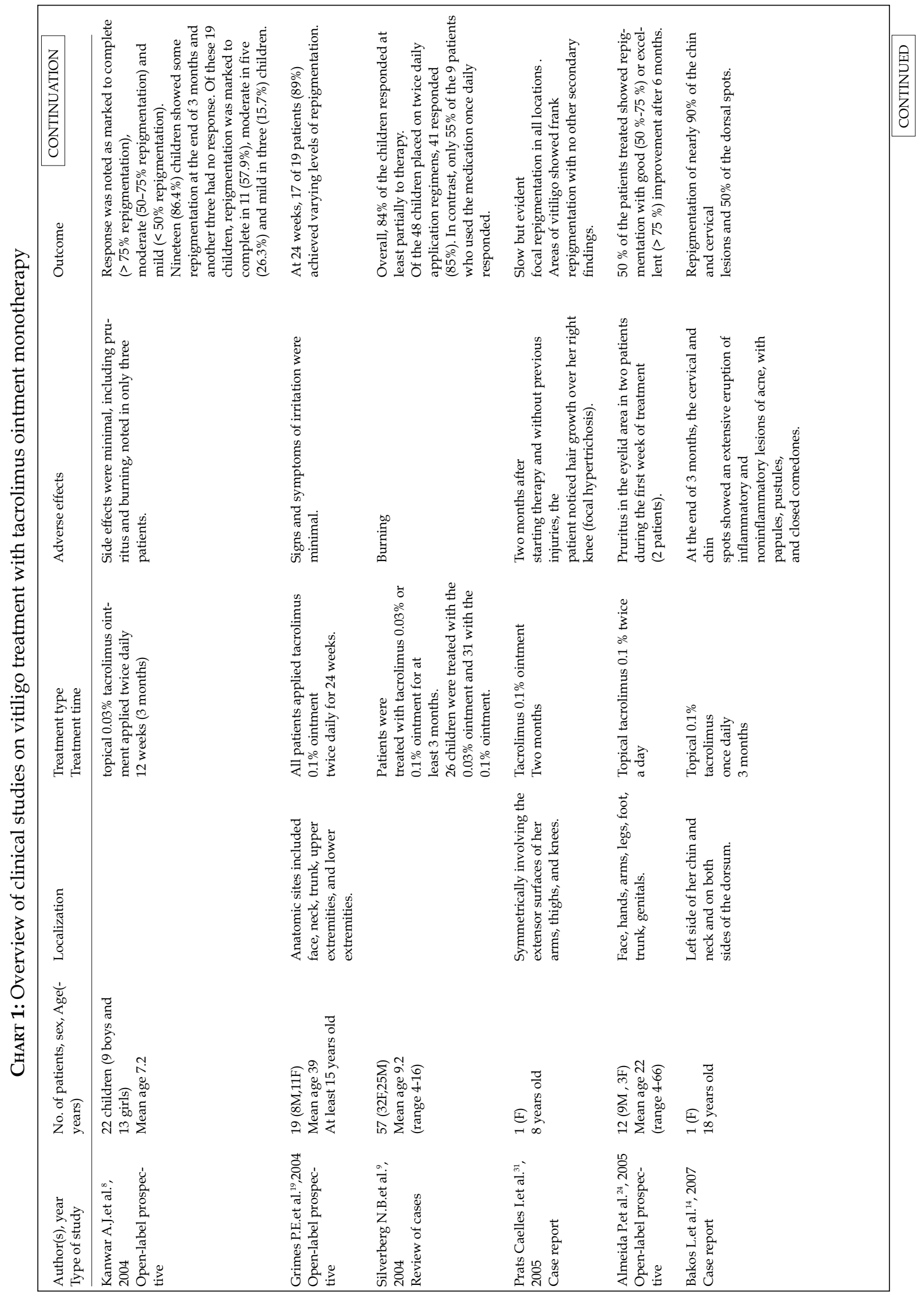




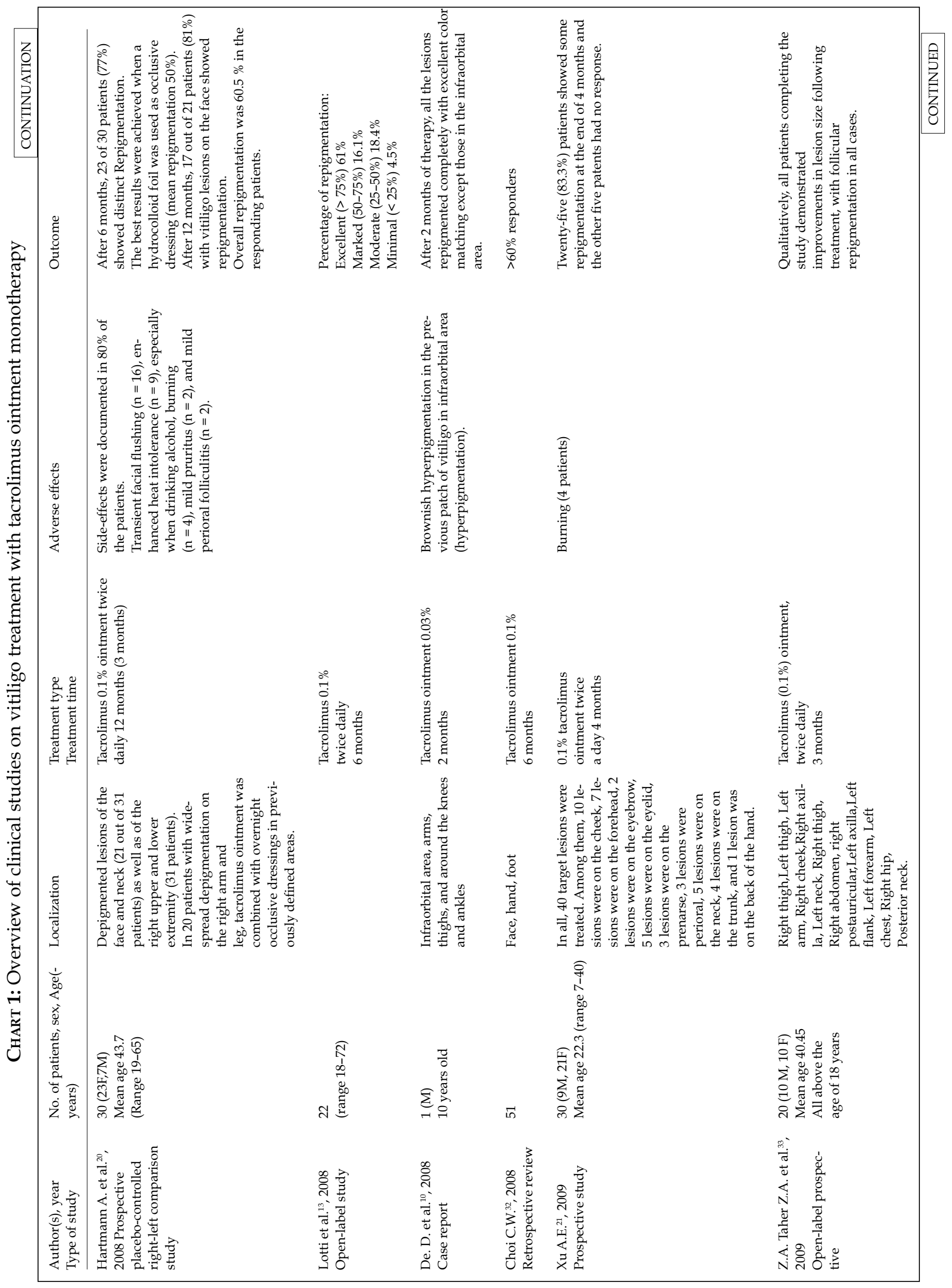



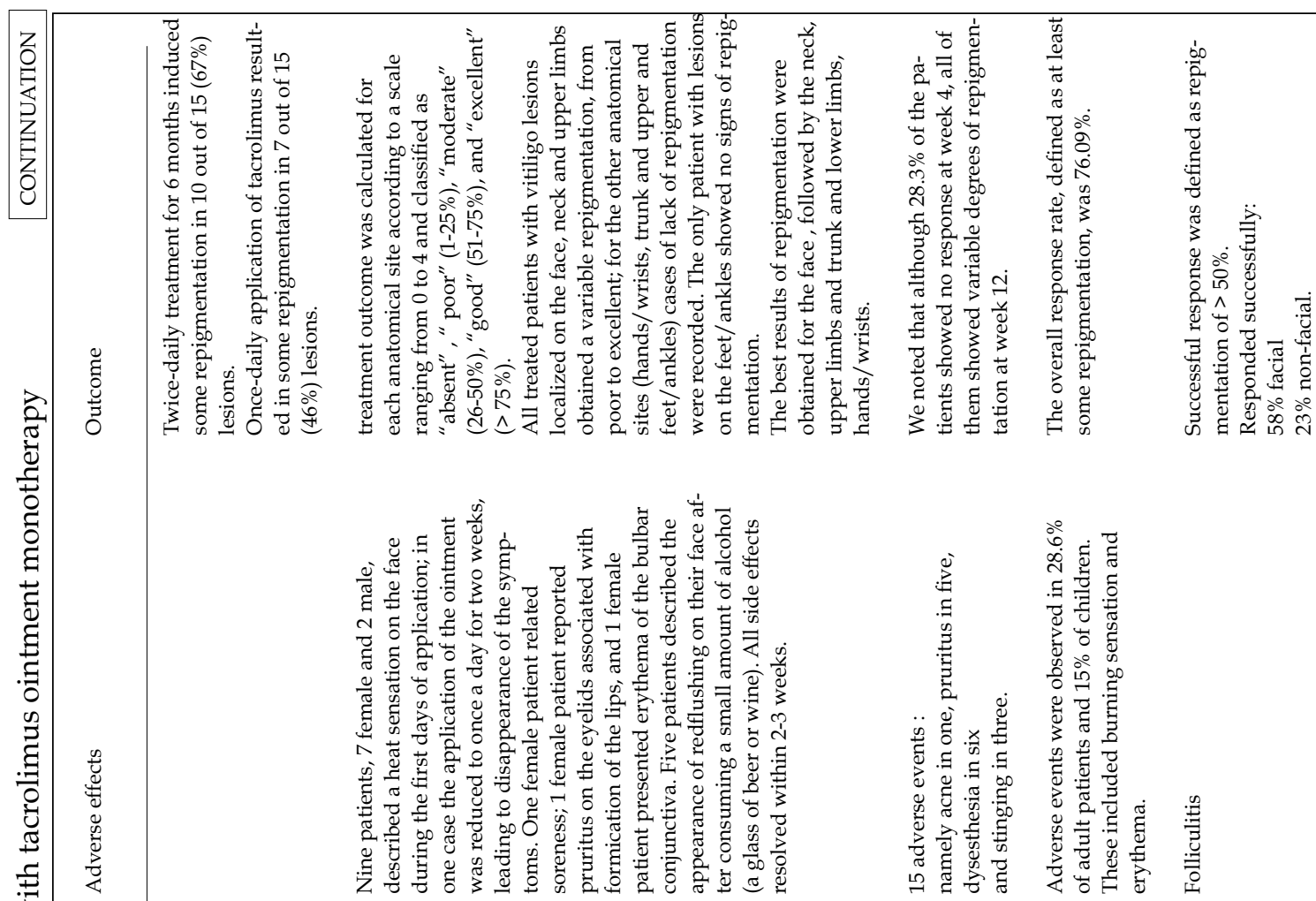

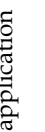

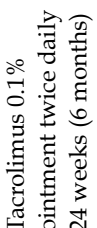

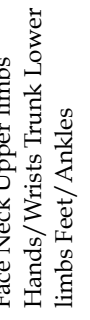

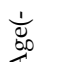

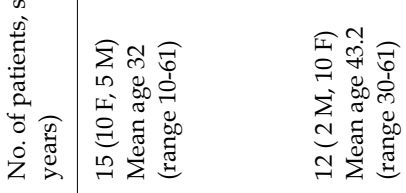

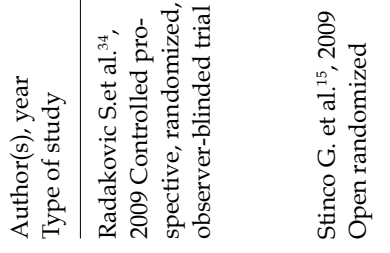
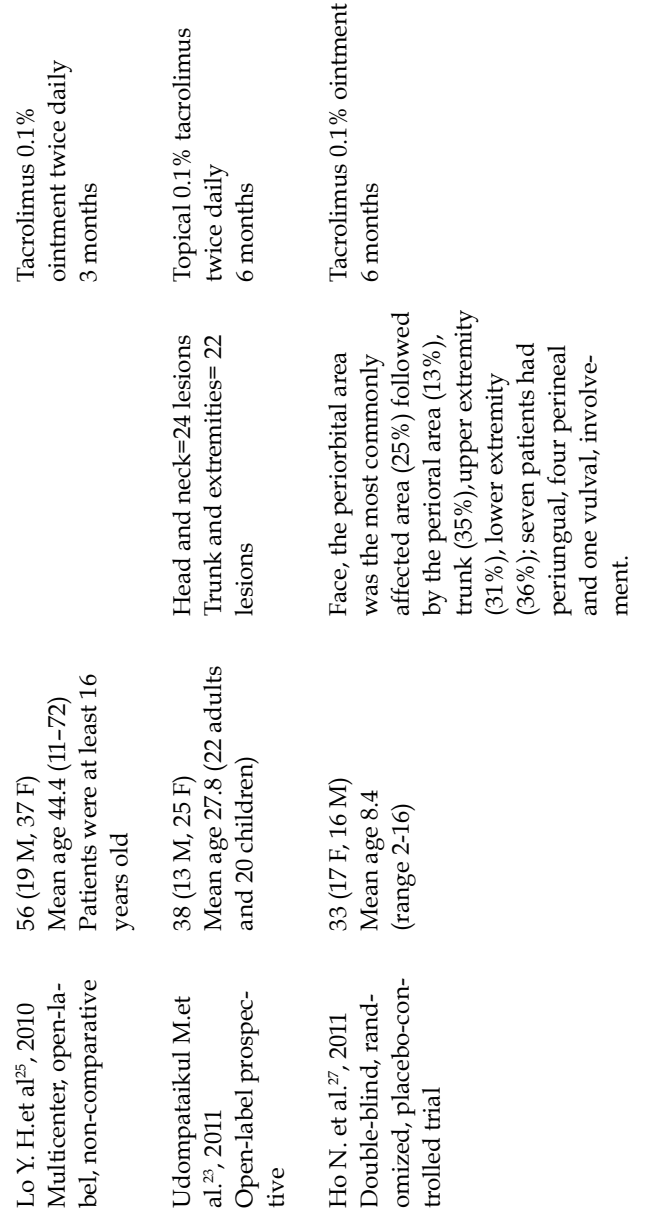


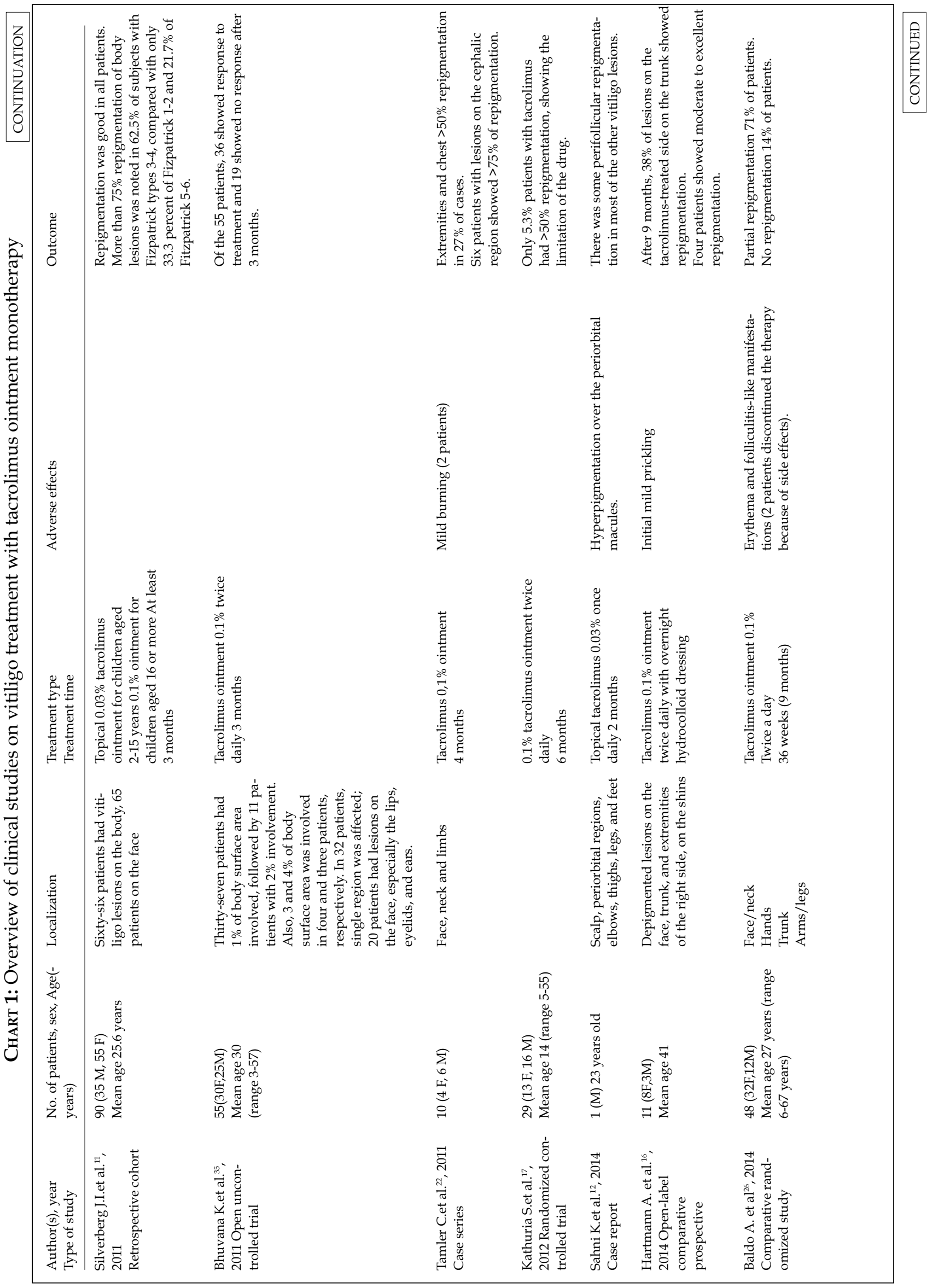


safe and free of major local side effects. No serious adverse events occurred that required treatment to be stopped. In a single study, erythema and folliculitis-like manifestations on the treated area led 2 patients to discontinue therapy with tacrolimus ointment $0.1 \%$ twice a day. ${ }^{26}$

In the open, randomized trial conducted by Stinco et al. ${ }^{15}$ (total of 12 patients, age range 30-61, treatment with tacrolimus $0.1 \%$ ointment twice daily), 9 patients described a heat sensation on the face during the first days of application. One female patient related the appearance of soreness; another female patient reported pruritus on the eyelids associated with formication of the lips, while one female patient presented erythema of the bulbar conjunctiva. Five patients described redflushing on their faces after consuming small amounts of alcohol (a glass of beer or wine). All local side effects resolved within 2-3 weeks after the topical treatment regimen was reduced from two daily applications to one daily application.
Tacrolimus $0.03 \%$ ointment has been reserved for children $<16$ years and associated with hyperpigmentation, as well as hypertricosis, in the target area. ${ }^{12,10,31}$ In In adult patients, treatment with tacrolimus $0.03 \%$ yielded similar results to tacrolimus ointment $0.1 \%$.

Although tacrolimus monotherapy seems to have good efficacy and tolerability, only small trials and case series are available in the literature.32,33,34,35 The largest study ever published in terms of the number of patients enrolled, only included 90 patients. ${ }^{11}$

Hence, further, standardized investigations on a greater number of patients are needed. 


\section{REFERENCES}

1. Allam M, Riad H. Concise review of recent studies in vitiligo. Qatar Med J. 2013;2013:1-19

2. Begum R, Marfatia YS, Laddha NC, Dwivedi M, Mansuri MS, Singh M. Vitiligo: a complex disease and a complex approach. Mol Cytogenet. 2014;7:I57.

3. Tarlé RG, Nascimento LM, Mira MT, Castro CC. Vitiligo - part 1. An Bras Dermatol. 2014;89:461-70.

4. Hengge UR. Off-label indications for topical tacrolimus. Hautarzt. 2013;64:752-6.

5. Wong R, Lin AN. Efficacy of topical calcineurin inhibitors in vitiligo. Int J Dermatol. 2013;52:491-6.

6. Lan CC, Chen GS, Chiou MH, Wu CS, Chang CH, Yu HS. FK506 promotes melanocyte and melanoblast growth and creates a favourable milieu for cell migration via keratinocytes: possible mechanisms of how tacrolimus ointment induces repigmentation in patients with vitiligo. Br J Dermatol. 2005;153:498-505.

7. Grimes PE, Soriano T, Dytoc MT. Topical tacrolimus for repigmentation of vitiligo. J J Am Acad Dermatol. 2002;47:789-91.

8. Kanwar AJ, Dogra S, Parsad D. Topical tacrolimus for treatment of childhood vitiligo in Asians. Clin Exp Dermatol. 2004;29:589-92.

9. Silverberg NB, Lin P, Travis L, Farley-Li J, Mancini AJ, Wagner AM, et al. Tacrolimus ointment promotes repigmentation of vitiligo in children: a review of 57 cases. J Am Acad Dermatol. 2004;51:760-6.

10. De D, Kanwar AJ. Tacrolimus-induced hyperpigmentation in a patch of vitiligo. Skinmed. 2008;7:93-4.

11. Silverberg Jl, Silverberg NB. Topical tacrolimus is more effective for treatment of vitiligo in patients of skin of color. Journal of drugs in dermatology : JDD 2011;10:507-10.

12. Sahni K, Gupta V, Khanna N. Hyperpigmentation in photo exposed patches of vitiligo following tacrolimus therapy. Indian Dermatol Online J. 2014;5:164-6.

13. Lotti T, Buggiani G, Troiano M, Assad GB, Delescluse J, De Giorgi V, et al. Targeted and combination treatments for vitiligo. Comparative evaluation of different current modalities in 458 subjects. Dermatol Ther. 2008;21:S20-6.

14. Bakos L, Bakos RM. Focal acne during topical tacrolimus therapy for vitiligo. Arch Dermatol. 2007;143:1223-4.

15. Stinco G, Piccirillo F, Forcione M, Valent F, Patrone P. An open randomized study to compare narrow band UVB, topical pimecrolimus and topical tacrolimus in the treatment of vitiligo. Eur J Dermatol. 2009;19:588-93.

16. Hartmann A, Lohberg L, Keikavoussi P, Eichner S, Schuler G. Treatment of Generalised Vitiligo with Tacrolimus $0.1 \%$ Ointment vs. UVB Intense Pulsed Light Phototherapy: A Pilot Study. Acta Derm Venereol. 2014;94:585-7.

17. Hartmann A, Löhberg L, Keikavoussi P, Eichner S, Schuler G. Segmental vitiligo: a randomized controlled trial to evaluate efficacy and safety of $0.1 \%$ tacrolimus ointment vs $0.05 \%$ fluticasone propionate cream. Acta Derm Venereol. 2014;94:585-7.

18. Lepe V, Moncada B, Castanedo-Cazares JP, Torres-Alvarez MB, Ortiz CA, TorresRubalcava $A B$. A double-blind randomized trial of $0.1 \%$ tacrolimus vs $0.05 \%$ clobetasol for the treatment of childhood vitiligo. Arch Dermatol. 2003;139:581-5.

19. Grimes PE, Morris R, Avaniss-Aghajani E, Soriano T, Meraz M, Metzger A. Topical tacrolimus therapy for vitiligo: therapeutic responses and skin messenger RNA expression of proinflammatory cytokines. J Am Acad Dermatol. 2004;51:52-61.

20. Hartmann A, Bröcker EB, Hamm H. Occlusive treatment enhances efficacy of tacrolimus $0.1 \%$ ointment in adult patients with vitiligo: results of a placebocontrolled 12-month prospective study. Acta Derm Venereol. 2008;88:474-9.

21. Xu AE, Zhang DM, Wei XD, Huang B, Lu LJ. Efficacy and safety of tarcrolimus cream $0.1 \%$ in the treatment of vitiligo. Int J Dermatol. 2009;48:86-90.

22. Tamler C, Duque-Estrada B, Oliveira PA, Avelleira JC. Tacrolimus $0,1 \%$ ointment in the treatment of vitiligo: a series of cases. An Bras Dermatol. 2011;86:169-72.

23. Udompataikul M, Boonsupthip P, Siriwattanagate R. Effectiveness of $0.1 \%$ topical tacrolimus in adult and children patients with vitiligo. J Dermatol. 2011;38:536-40.

24. Almeida P, Borrego L, Rodríguez-López J, Luján D, Cameselle D, Hernández B. Vitiligo. Treatment of 12 cases with topical tacrolimus. Actas Dermosifiliogr. 2005;96:159-63.

25. Lo YH, Cheng GS, Huang CC, Chang WY, Wu CS. Efficacy and safety of topical tacrolimus for the treatment of face and neck vitiligo. J Dermatol. 2010;37:125-9.

26. Baldo A, Lodi G, Di Caterino P, Monfrecola G. Vitiligo, NB-UVB and tacrolimus: our experience in Naples. G Ital Dermatol Venereol. 2014;149:123-30.

27. Ho N, Pope E, Weinstein M, Greenberg S, Webster C, Krafchik BR. A double-blind, randomized, placebo-controlled trial of topical tacrolimus $0.1 \%$ vs. clobetasol propionate $0.05 \%$ in childhood vitiligo. Br J Dermatol. 2011;165:626-32.
28. Smith DA, Tofte SJ, Hanifin JM. Repigmentation of vitiligo with topical tacrolimus. Dermatology. 2002;205:301-3.

29. Travis LB, Weinberg JM, Silverberg NB. Successful treatment of vitiligo with $0.1 \%$ tacrolimus ointment. Arch Dermatol. 2003;139:571-4.

30. Tanghetti EA. Tacrolimus ointment $0.1 \%$ produces repigmentation in patients with vitiligo: results of a prospective patient series. Cutis. 2003;71:158-62.

31. Prats Caelles I, Herranz Pinto P, de Ayala Casado EL, de Lucas Laguna R. Focal hypertrichosis during topical tacrolimus therapy for childhood vitiligo. Pediatr Dermatol. 2005;22:86-7.

32. Choi CW, Chang SE, BakH, Choi JH, Park HS, Huh CH, et al. Topical immunomodulators are effective for treatment of vitiligo. J Dermatol. 2008;35:503-7.

33. Taher ZA, Lauzon G, Maguiness S, Dytoc MT. Analysis of interleukin-10 levels in lesions of vitiligo following treatment with topical tacrolimus. $\mathrm{Br} \mathrm{J}$ Dermatol. 2009;161:654-9.

34. Radakovic S, Breier-Maly J, Konschitzky R, Kittler H, Sator P, Hoenigsmann H, et al. Response of vitiligo to once- vs. twice-daily topical tacrolimus: a controlled prospective, randomized, observer-blinded trial. J Eur Acad Dermatol Venereol. 2009;23:951-3.

35. Bhuvana K, Sarala N, Singh G, Kumar TN. Effect of $0.1 \%$ tacrolimus ointment in localized vitiligo: an open uncontrolled trial. Indian J Dermatol. 2011;56:445-6.

\author{
MAILING ADDRESS: \\ Andrea Sisti \\ Via Venezia 6 \\ 51016 \\ Montecatini Terme, Pistoia, Italy \\ phone: 0039-3477493002 \\ E-mail: asisti6@gmail.com
}

How to cite this article: Sisti A, Sisti G, Oranges CM. Effectiveness and safety of topical tacrolimus monotherapy for repigmentation in vitiligo: a comprehensive literature review. An Bras Dermatol. 2016;91(2):187-95. 